\title{
The Correlation between Mothers with Low Birth Weight History and Body Mass Index Leptin Levels, and Preeclampsia
}

\author{
Aryani Aziz*, Johanes C. Mose, Anita Deborah \\ Department of Obstetrics and Gynecology, Faculty of Medicine, Padjajaran University/Hasan Sadikin Hospital, \\ Bandung, Indonesia \\ Email: "aryaniaziz88@gmail.com
}

Received 28 November 2015; accepted 17 December 2015; published 21 December 2015

Copyright (C) 2015 by authors and OALib.

This work is licensed under the Creative Commons Attribution International License (CC BY). http://creativecommons.org/licenses/by/4.0/

(c) (i) Open Access

\section{Abstract}

Preeclampsia (PE) is one of the complications of pregnancythat significantly increases maternal and fetal mortality and morbidity. Mothers who were born as low birth weight (LBW) babies have risks for PE that are 2 - 3 times higher than maternal birth weight $\geq 2500$ g. LBW history of the mother is also correlates with the incidence of metabolic syndrome later in life including incresed of body mass index (BMI), hyperlipidemia, hypertension, stroke type 2 diabetes and cardiovascular accident (CVA). This study aimed at assessing the correlation between the incidence of LBW history of the mother with PE, BMI and leptin levels. This study was a retrospective study of maternal LBW history with case control design as well as cross-sectional design to search the correlation between BMI and leptin levels with the incidence of PE. This study was conducted from December 2014 through February 2015 in Hasan Sadikin Hospital Bandung. Data were analyzed using Spearman Rank and Chi-Square, and the significant difference was determined as $\alpha<0.05$. The results showed that maternal LBW history correlated with an increased in systolic $(r=-0.417 ; p=0.017)$ and diastolic $(r=-0.498 ; p=0.004)$ blood pressure but did not correlated with the incidence of PE $(r=0.333 ; p=0.127)$. The incidence of maternal history of LBW was higher in cases of PE ( $p=$ 0.033). There was a correlation between LBW history and early onset preeclampsia (EOPE) and late onset preeclampsia (LOPE) $(r=0.500 ; p=0.048)$. There was no correlation between LBW history of BMI $(r=-0.040 ; p=0.830)$ and levels of leptin $(r=-0.157 ; p=0.390)$. There was a correlation of BMI and leptin levels ( $r=0.664 ; p=0.0001)$ Maternal LBW history was correlated with increased incidence of systolic and diastolic blood pressure and the incidence of EOPE and LOPE.

\section{Keywords}

Body Mass Index, Mother, Early Onset Preeclampsia, Late Onset Preeclampsia, Leptin Levels, Low Birth Weight

${ }^{*}$ Corresponding author.

How to cite this paper: Aziz, A., Mose, J.C. and Deborah, A. (2015) The Correlation between Mothers with Low Birth Weight History and Body Mass Index Leptin Levels, and Preeclampsia. Open Access Library Journal, 2: e2085.

http://dx.doi.org/10.4236/oalib.1102085 


\section{Introduction}

The phenomenon of low birth weight babies (LBW) is a due to problem in the health sector. World Health Organization (WHO) defines that LBW infants are impaired growth in the womb leading to birth weight $<2500$ gram [1]-[3]. Epidemiological data show that morbidity and mortality in LBW group numbers continue to rise significantly. Several studies in developing countries reported contributing of LBW to low birth weight infant morbidity and mortality is quite large. LBW figures compiled by a team of health survey in 62 countries expressed the incidence of low birth Weight in the range of 3\% to $12.5 \%$ [4]-[6].

LBW condition occurs due to pregnancy for preterm and growth retardation (IUGR) [6]-[8]. In the pathophysiology LBW, aspects related to the mother, placenta and fetus to form an interlocking mechanism. In addition to these three aspects, genetic and environmental factors may also affect the phenotype babies born.

Several etiology and pathogenesis have been proposed as the etiology and pathogenesis of preeclampsia. Some of the theories include ischemia of the placenta, prostacycline (PGI2) and thromboxane (TXA2) involvement, immune maladaptation, oxidative stress, genetic, nutritional factor and endothel dysfunction.

Barker research shows that in general LBW can be caused by environmental conditions in the uterus are less supportive of infant development process. LBW as a consequence of the adaptation process in the womb has been categorized as a risk factor for the onset of metabolic syndrome, such as hypertension, type 2 diabetes, cardiovascular disease (CVD) such as chronic heart disease (CHD) and stroke.

The incidence of severe PE can be characterized by leakage of capillaries (proteinuria, ascites, and pulmonary edema), weight gain $>5 \mathrm{lb} /$ week, or an imbalance in the body homeostasis with multiorgan dysfunction. Patients in this condition experience clinical manifestations as PE atypical that were consistent with abnormal laboratory tests or in the form of observed symptoms [9].

Preeclampsia is diagnosed clinically after the 20th week of pregnancy or within the first 4 - 6 weeks postpartum [9]. Incidence of preeclampsia causes 76,000 maternal deaths and 500,000 infant deaths per year. Clinical signs and symptoms of severe PE include high blood pressure or hypertension, proteinuria, increased body weight in the extreme and sudden, headaches, swelling, and blurred eyesight.

Preeclampsia can occur $\geq 34$ weeks pregnancy or can be called a late onset preeclampsia (LOPE) [10]-[13]. However, approximately $10 \%$ of cases of PE occur $<34$ week of pregnancy so-called early-onset preeclampsia (EOPE). Placentation process interrupted in the early implantation is a crucial point that distinguishes the pathophysiology of EOPE and LOPE. At EOPE disorders that occur during early implantation in the process of trophoblast invasion and spiral artery remodelling, while LOPE occurs when maternal syndrome appeared in $\geq 34$ weeks gestation due to dysfunction and dysregulation of maternal tolerance against inflammation. EOPE condition is considered more like a disease as a disease of the placenta while LOPE is like a disease of maternal [14] [15].

Sign and symptoms of preeclampsia are varied in each patient. In general, preeclamsia showed some symptoms such as hypertensive dysorder (systolic blood pressure $\geq 140 \mathrm{mmHg}$ or dyastolic blood pressure $\geq 90$ $\mathrm{mmHg}$ ), Proteinuria of $300 \mathrm{mg}-5 \mathrm{~g}$ in 24 hours in more than 20 weeks of gestational age. Other symptoms that can appear are systolic blood preesure $>160 \mathrm{mmHg}$ or dyastolic blood pressure $>110 \mathrm{mmHg}$, increase creatinine level, oligouria, epigastric pain, persistent headache, blurred vision, lung oedema, increase of Aspartate Transaminase (AST) and Alanine Transaminase (ALT), thrombocytopenia, and eclampsia.

A number of studies have reported that serum leptin plays a role in the pathogenesis of PE, so that an increase in leptin levels may help prediction of disease and a marker of the severity of PE. Serum Leptin is also known to play a significant role as a biomarker of EOPE and LOPE in relation to the severity of the disease, body mass index (BMI), IUGR and LBW [16]-[18]. Low birth weight and BMI are known to correlate with risk factors for cardiovascular disease. The mothers were born as low birth weight and then obese in adulthood have the highest risk factor profile. This profile includes characteristics such as hyperlipidemia metabolic syndrome that appear in the form of PE [19]-[21].

This study aimed to analyze the relationship between mother with LBW history, BMI, leptin levels and the incidence of PE. The incidence of PE is still high to be the reason of writing this study so that it is expected 
there is some alternative measures to reduce the number ofmorbidity and mortality of mothers and infants.

\section{Methods}

Subjects of this study were patients with PE study group, and normal pregnant patients as control group who came to the Department of Obstetrics and Gynecology, Faculty of Medicine/Government General Hospital of Dr. Hasan Sadikin, Bandung who met the inclusion the exclusion criteria. A number of subjects have to fill out an informed consent as evidence of willingness to follow a series of research process. Correlational analysis was conducted to examine the relationship between the variables presented through data analysis. Determination of sample size in this study is consistent with the purpose of research that is by using the formula determining the sample size for the study of numerical correlation analysis. Thus the minimum required number of samples is equal to at least 29 samples.

Total subjects involved in this research consisted of 125 patients with normal pregnancy and 40 patients withPE at 20 - 40 weeks gestational age who gave birth in Government General Hospital Dr. Hasan Sadikin Bandung from December 2014 to February 2015.

After filtering through inclusion and exclusion criteria, 16 preeclampsia (PE) patients declared eligible for analysis. As for normal pregnant found as many as 16 patients after matching with the patient PE based on confounding factors. 32 samples were interviewed through a questionnaire to find more detailed information about the history of mother with LBW history.

A retrospective study was conducted on maternal LBW history with case-control design and cross sectional study, as the case group was the mother with PE meanwhile the control group was normal pregnant mothers. Observational studies and correlation analysis were conducted to find the relationship between variables using cross sectional design to search the correlation of BMI and leptin levels. Univariate analysis of the data on categorical variables was used to see the proportions of each variable to be presented descriptively.

Statistical methods used to analyzed the data are using Rank Spearman test, lambda, Mann whitney test, chi square, Fisher Exact test and independent sample t-test to gained $p$-value. The standard of significance level or $p$ value is $5 \%$.

\section{Results}

Subjects in this study were patients severePE and normal pregnancy. Total subjects were assessed based on height and weight to determine BMI. Then, taking as much as 3 cc of blood was done to examine leptin levels for analyzing the blood pressure and its relation to obesity. This research was conducted in the Department of Obstetrics and Gynecology, Faculty of Medicine/Government General Hospital Dr. Hasan Sadikin, Bandung.

Based on the characteristics of the test variables as age, parity, and maternal gestational age at birth, the results showed a $p$-value greater than $0.05(p>0.05)$ (Table 1). It described the two groups do not have different characteristics. All characteristics variables were declared similar or homogeneous, then the two groups deserved to be compared and analyze.

LBW condition occurs due to preterm pregnancy and full-term in stunted fetal growth. This study found maternal gestational age at birth in normal pregnancy and severe PE was a full-term pregnancy that each was 14 persons (87.5\%) and preterm pregnancy in total was 2 (12.5\%). Values obtained through different test showed a non-significant values where $p=1.0(p>0.05)$ (Table 1$)$.

Lambda correlation test was performed to analyze the data category with a nominal par between the two variables that are not equivalent (Table 2). Lambda correlation test for normal women with a LBW history and PE showed a $p$ value of $0.127(p>0.05)$. The value obtained is not significant so that there is no correlation between the incidence of LBW mother with PE.

The relationship between a history of LBW with systolic blood pressure were analyzed statistically. The analysis showed a $p$ value of $0.017(p<0.05)$ and was declared statistically significant (Table 3). Based on the values obtained, there is a correlation between maternal LBW history and systolic blood pressure. Coefficient value of correlation states toward a negative correlation with the strength of the correlation is strong enough (moderate).

Results of the analysis of the relationship of the mother's history of LBW and diastolic blood pressure showed a $p$-value of $0.004(p<0.05)$ (Table 3). Results of the study revealed a relationship between the two variables were statistically significant. Therefore, there is a correlation between maternal LBW history and diastolic blood 
pressure.

Test comparison of the proportion of mothers with LBW and severe PE incident showed a $p$ value of 0.033 ( $p$ $<0.05$ ) and was statistically significant (Table 4).

Research results shows there are differences statistically significant proportion between a mother with a history of low birth and incidence of severe PE.

Correlation between maternal with LBW history in the group of EOPE andLOPE and the gestation was analyzed. Results of analysis was a $p$-value $0.048(p<0.05)$ and was statistically significant (Table 5). These findings indicate that there is a correlation among the incidence of maternal with LBW history and EOPE and LOPE.

Table 1. Subjects characteristics.

\begin{tabular}{|cccc|}
\hline Variabel & Normal $(\mathbf{n}=\mathbf{1 6})$ & Preeclampsia (n = 16) & $\boldsymbol{p}$ \\
\hline Maternal Age & & & \\
\hline Mean \pm SD & $27.63 \pm 6.12$ & $30.31 \pm 7.43$ & $\mathbf{0 . 2 7 3}$ \\
Median & 26.50 & 30.50 & \\
Range & 22.00 & 24.00 & \\
\hline Parity & & & $\mathbf{0 . 1 1 3}$ \\
\hline Primipara & $14(87.5 \%)$ & $9(56.2 \%)$ & \\
Multipara & $2(12.5 \%)$ & & $\mathbf{1 . 0 0 0}$ \\
\hline Mother's gestational age at birth & & $2(12.5 \%)$ & $14(87.5 \%)$ \\
\hline Preterm & $2(12.5 \%)$ & $14(87.5 \%)$ & \\
\hline Term
\end{tabular}

Notes: $p$ value was analyzed using Chi Square and Exact Fisher. For numerical data, $p$ value was analyzed with independent t-test if the data distribution is normal, if the data distribution is not normal, mannwhtiney test was used.

Table 2. Correlation between low birth weight history mother and preeclampsia.

\begin{tabular}{ccccc}
\hline LBW & Normal $(\mathbf{n}=\mathbf{1 6})$ & Preeclampsia $(\mathbf{n}=\mathbf{1 6})$ & $\boldsymbol{r}$ & $\boldsymbol{p}$ value \\
\hline Yes & $4(25 \%)$ & $10(62.5 \%)$ & $\mathbf{0 . 3 3 3}$ & $\mathbf{0 . 1 2 7}$ \\
No & $12(75 \%)$ & $6(37.5 \%)$ & & \\
\hline
\end{tabular}

Notes: $p$ value was analyzed with Lambda correlation test.

Table 3. Correlation of low birth weight history mother with systolic and diastolic blood pressure.

\begin{tabular}{ccc} 
Correlation & $\boldsymbol{r}$ & $\boldsymbol{p}$ value \\
\hline Mother's birth weight to systolic blood pressure & -0.417 & $0.017^{* *}$ \\
Mother's birth weight to diastolic blood pressure & -0.498 & $0.004^{* *}$ \\
\hline
\end{tabular}

Notes: the correlation between the numerical and ordinal using spearmann rank correlation analysis.

Table 4. Comparison of proportion of low birth weight history in preeclampsia.

\begin{tabular}{cccc}
\hline LBW & Normal $(\mathbf{n}=\mathbf{1 6})$ & Preeclampsia $(\mathbf{n}=\mathbf{1 6})$ & Nilai $\boldsymbol{p}$ \\
\hline Yes & $4(25 \%)$ & $10(62.5 \%)$ & $\mathbf{0 . 0 3 3}^{* *}$ \\
No & $12(75 \%)$ & $6(37.5 \%)$ & \\
\hline
\end{tabular}

Notes: For categorical data, $p$-value is calculated based on chi square test.

Table 5. Correlation of mother with LBW history and EOPE, LOPE, BMI and leptin levels.

\begin{tabular}{ccc}
\hline Criteria & r & p value \\
\hline LWB with EOPE and LOPE & $0.500^{*}$ & $0.048^{* *}$ \\
LBW with BMI & -0.0 .40 & 0.830 \\
LBW with Leptin Levels & -0.157 & 0.390 \\
\hline
\end{tabular}

Notes: The correlation between numerical and ordinal using spearman rank correlation analysis. 
$P$-values were obtained $0.830(\mathrm{p}>0.05)$ that is expressed not statistically significant. Based on the research results, there is no correlation between LBW history mothers and BMI

Based on Figure 1 shows there is a positive correlation with the strength of a moderate correlation between mothers with LBW and gestational age. Thus the lower of the birth weight history, the EOPEPE occurs. In this study it can be concluded that the EOPE incident occurred at the gestational age less than 33 weeks.

Analysis of correlation between the BMI patients and high levels of leptin obtains $p$-value $0.0001(p<0.05)$ and was statistically significant (Table 6). These findings reveal that there is a correlation between BMI and high levels of leptin in the direction of a positive correlation with the strength of a strong correlation between BMI and leptin levels with r: 0664.

\section{Discussion}

Age of the mother during pregnancy affects the outcome of pregnancy. The statement was supported by the data released by the American College of Obstetricians and Gynecologists (ACOG), which revealed that pregnancy in adolescents aged 15 - 19 reaches $11 \%$ of total pregnancies in the United State [22]. Adolescents are more likely to develop complications of anemia, IUGR, prematurity, neonatal death, and even sexually transmitted diseases. Moreover, pregnancy at the age of 35 years or show a tendency for the onset of medical complications such as gestational diabetes, hypertension in pregnancy, placenta previa, placental abruption and delivery risks with section sesarea [23].

Based on the data processed, the study found primiparous pregnancy in 14 normal pregnancies and the PE as many as 9 people or more numerous than multiparous pregnancy that is 2 in normal pregnancy and 7 in PE (Table 1). This is consistent with the theory that states that the pathophysiology of preeclampsia primigravid is the cause of preeclampsia occurrence, two-thirds of cases occur in the first pregnancy that took place since the first trimester, although the risk is the same both in primigravida and multigravida [24]. Primigravid with very young age occurs because the patients rarely checkups, hide her pregnancy, and ignore of the importance of antenatal.

Correlation incidence of mothers with LBW history and PE is not significant, but when the correlation of the incidence of mother with LBW history connected to systolic and diastolic blood pressure showed statistically significant or meaningful (Table 2 and Table 3).

Comparative analysis of the proportion of mothers with a history of LBW and severe PE were statistically significant (Table 4). The study states that the incidence of maternal history of LBW may be a risk factor for PE occurrence. A study explains that LBW in general can be caused by environmental conditions in the uterus are less supportive of the baby development process [25]. Furthermore, the study also explains that babies are born as LBW tend to be susceptible to metabolic disorders or metabolic syndrome as a vascular system and hormonal including the occurrence of hypertension in pregnancy.

One study found mothers who were born as LBW have a 70\% risk of experiencing hypertension in pregnancy than a mother who was born a normal birth weight babies [15]. Mothers who were born weighing $<4.5$ pounds, has the highest risk for PE when compared with mothers who were born weighing $\geq 8.5$ pounds [26]. Other studies have reported 80 studies that describe the relationship between blood pressure of adults and a history of birth weight found that the two have a relationship that is inversely proportional [27].

Analysis of the correlation between maternal of LBW history in the group of EOPE and LOPE, and gestational age showed statistically significant value. Based on the values obtained, there is a positive correlation with the strength of the correlation is strong enough (moderate) in the incidence of mother with LBWhistory in the group EOPEand LOPE, and gestational age (Table 5). It shows that the lower LBW history, the EOPE occurs. This study reveals that EOPE occurred at the gestational age $<33$ weeks (Figure 1).

Results obtained through statistical analysis showed the incidence of EOPE occured earlier. The findings did not fit when compared to previous studies that said that the EOPE occured in pregnancy < 34 week [10]-[13]. Shallow trophoblast invasion and spiral artery remodeling disorder is a fundamental pathophysiology of the EOPE. At EOPE disorders occur during early implantation while at LOPE disorders occur when maternal syndrome appeared in $\geq 34$ weeks gestation due to dysfunction and dysregulation of maternal tolerance against inflammation.

Correlation analysis to women with a history of LBW andBMI is not statistically significant (Table 5). Based on the results obtained, there is no correlation between the incidence of maternal history of LBWand BMI. These findings are not consistent with previous research that states LBW and BMI were correlated with risk 


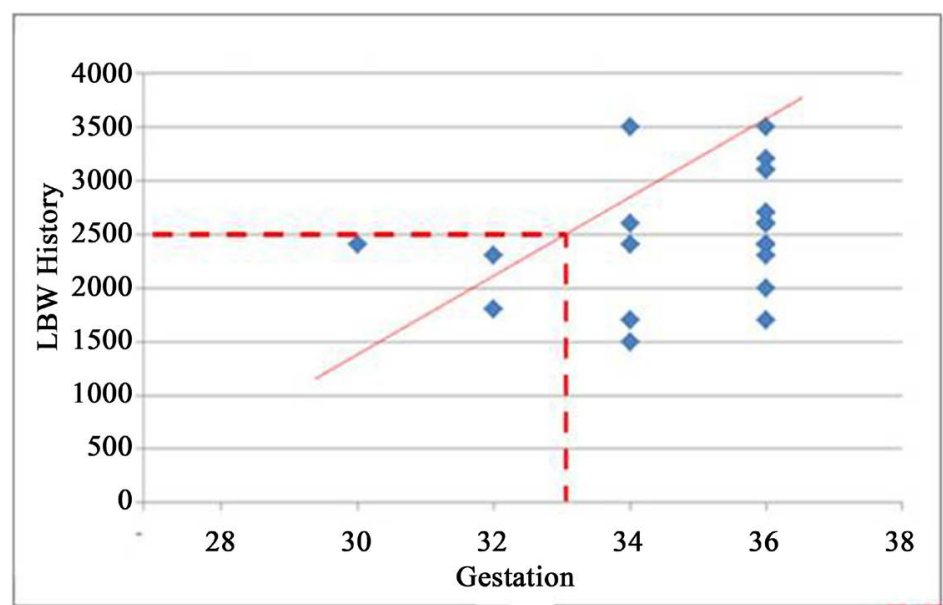

Figure 1. Correlation of LBW history mother and EOPE and LOPE.

\section{Table 6. Correlation between BMI and leptin levels.}

\begin{tabular}{ccc} 
Correlation & $\boldsymbol{R}$ & Nilai $\boldsymbol{p}$ \\
\hline BMI and Leptin Levels & $-0.664^{*}$ & $0.0001^{* *}$ \\
\hline
\end{tabular}

Notes: Spearman correlation is used because the data is not normally distributed.

factors CVD [19]. Skinny mothers who were born as LBW have a two times higher risk for PE than women with normal BMI, but the obesity mothers with a history born as LBW have a four times higher risk for PE. The mothers were born as LBW and then obese in adulthood have the highest risk factor profile. This profile includes characteristics such as hyperlipidemia metabolic syndrome that appear in the form of PE.

Numerous studies have examined the influence of maternal birth weight on the risk of PE as a precursor of chronic diseases in the future [9] [19] [28]. In the study states PE risk decreased with increasing maternal birth weight. Mothers who have a history of LBW are known to have 2 - 3 times higher risk to experience PE than women with a history of birth weight $2500-2999$ grams. Mothers with a history $\geq 4.000$ grams birth weight appear to have decreased the risk of PE [28].

Results of the study that stated insignificant is caused by taking the mothers' weight while inpartu not weight before pregnancy so that BMI examined was inaccurate. In 1990, it was issued a reference table as the recommended weight gain for every group of BMI before pregnancy.

Results of correlation analysis of LBW mothers with higher levels of leptin showed that a value is not statistically significant (Table 5). Based on the results obtained, there is no correlation between a history of LBW mothers with higher levels of leptin. This is not in line with research that revealed leptin may influence the production of Nitric Oxide and natriuresis, causing sodium retention, systemic vasoconstriction, and increased blood pressure [29]. In the study, leptin plays a role in the pathogenesis of hypertension in obese patients. Another study describes the serum leptin levels increased in the PE group compared with the normal group [30]. Leptin pregnancy serum biomarkersis known to play a significant role as EOPE and LOPE in relation to the Severity of the disease, BMI, IUGR and LBW [31] [32].

Results of the analysis between BMI andleptin levels by using correlation testwere statistically significant (Table 6). This indicates that there is a correlation between BMI and high levels of leptin in the direction of a positive correlation with the strength of a strong correlation between BMI and high levels ofleptin.

\section{Conclusions}

There is no positive correlation between the incidence of maternal with LBW history andPE but positive correlation between the incidence of maternal with LBW history and Revalon of EOPE and LOPE. There is no correlation between mother with a history of LBWand either BMI or leptin levels. There is negative correlation among maternal with LBW history and systolic and diastolic blood pressure.

This study also shows that the incidence of maternal history of LBW are more common in cases of PE and 
positive correlation between BMI and leptin levels on the incidence of PE.

In the future, preventive measures to prevent or detect preeclampsia such as using doppler on uterine artery during antenatal care below 20 weeks of gestational age should be done on evey patient. Extra precaution to mother with history of preeclampsia should be monitored carefully.

\section{References}

[1] Kramer, M. (1987) Determinants of Low Birth Weight: Methodological Assessment and Metaanalysis. Bull WHO, 65, 663-737.

[2] UNICEF-WHO (2004) Low Birth Weight: Country, Regional, and Global Estimates. 1-31.

[3] Chen, Y., Li, G., Ruan, Y., Zou, L., Wang, X. and Zhang, W. (2013) An Epidemiological Survey on Low Birth Weight Infants in China and Analysis of Outcomes of Full-Term Low Birth Weight Infants. BMC Pregnancy and Childbirth, 13, 242. http://dx.doi.org/10.1186/1471-2393-13-242

[4] BPS (2002) End Decade Statistical Report: Data and Descriptive Analysis Jakarta Biro Pusat Statistik.

[5] Hediger, M.L., Overpeck, M.D., Maurer, K.R., Kuczmarski, R.J., McGlynn, A. and Davis, W.W. (1998) Growth of Infants and Young Children Born Small or Large for Gestational Age: Findings from the Third National Health and Nutrition Examination Survey. Archives of Pediatrics and Adolescent Medicine, 152, 1225-1231. http://dx.doi.org/10.1001/archpedi.152.12.1225

[6] Le Roith, D., Bondy, Y.N., Yakar, S., Liu, J. and Buttler, A. (2001) The Somatomedin Hypothesis. Endocrine Reviews, 22, 53-74. http://dx.doi.org/10.1210/edrv.22.1.0419

[7] Ford, G.W., Doyle, L.W., Davis, N.M. and Callanan, C. (2000) Very Low Birth Weight and Growth into Adolescence. Archives of Pediatrics and Adolescent Medicine, 154, 778-784. http://dx.doi.org/10.1001/archpedi.154.8.778

[8] Black, L., Allen, Z., Bhutta, L., Caulfield, M., de Onis, M., Ezzati, C., et al. (2008) Maternal and Child Undernutrition: Global and Regional Exposures and Health Consequences. The Lancet, 371, 243-260. http://dx.doi.org/10.1016/S0140-6736(07)61690-0

[9] Sibai, B. and Stella, C. (2009) Diagnosis and Management of Atypical Preeclampsia-Eclampsia. American Journal of Obstetrics and Gynecology, 200, e1-e7.

[10] Laskowska, M., Laskowska, K., Terbosh, M. and Oleszczuk, J. (2013) A Comparison of Maternal Serum Levels of Endothelial Nitric Oxide Synthase, Asymmetric Dimethylarginine, and Homocysteine in Normal and Preeclamptic Pregnancies. Medical Science Monitor, 19, 430-437. http://dx.doi.org/10.12659/MSM.883932

[11] Ogge, G., Chaiworapongsa, T., Romero, R., Hussein, Y., Kusanovic, J.P., Yeo, L., et al. (2011) Placental Lesions Associated with Maternal Underperfusion Are More Frequent in Early-Onset than in Late-Onset Preeclampsia. Journal of Perinatal Medicine, 39, 641-652. http://dx.doi.org/10.1515/jpm.2011.098

[12] Redman, C.W.G. and Sargent, I.L. (2005) Latest Advances in Understanding Preeclampsia. Science, 308, $1592-1594$. http://dx.doi.org/10.1126/science.1111726

[13] Sanjay, G. and Girija, W. (2014) Preeclampsia-Eclampsia. The Journal of Obstetrics and Gynecology of India, 64, 413. http://dx.doi.org/10.1007/s13224-014-0502-y

[14] Grill, S., Rusterholz, C., Zanetti-Dällenbach, R., Tercanli, S., Holzgreve, W., Hahn, S., et al. (2009) Potential Markers of Preeclampsia-A Review. Reproductive Biology and Endocrinology, 7, 1-14. http://dx.doi.org/10.1186/1477-7827-7-70

[15] Villa, P.M., Hämäläinen, E., Mäki, A., Räikkönen, K., Pesonen, A.K., Taipale, P., et al. (2013) Vasoactive Agents for the Prediction of Early- and Late-Onset Preeclampsia in a High-Risk Cohort. BMC Pregnancy and Childbirth, 13, 1-10. http://dx.doi.org/10.1186/1471-2393-13-110

[16] Salimi, S., Farajian-Mashhadi, F., Naghavi, A., Mokhtari, M., Shahrakipour, M., Saravani, M., et al. (2014) Different Profile of Serum Leptin between Early Onset and Late Onset Preeclampsia. Hindawi Publishing Corporation Disease Markers, 2014, 1-8.

[17] Michael, A., Mary, Z., Christine, O. and Christos, M. (2004) Increased Leptin Levels in Preeclampsia: Associations with BMI, Estrogen, and SHBG Levels. Hormones, 3, 46-52. http://dx.doi.org/10.14310/horm.2002.11111

[18] Sumawan, H., Purwara, B.H. and Krisnadi, S.R. (2013) Low Maternal Leptin Levels in Preeclamptic Women with Fetal Growth Restriction. Open Journal of Obstetrics and Gynecology, 3, 536-540. http://dx.doi.org/10.4236/ojog.2013.37096

[19] Dempsey, J.C., Williams, M.A., Luthy, D.A., Emanuel, I. and Shy, K. (2003) Weight at Birth and Subsequent Risk of Preeclampsia as an Adult. American Journal of Obstetrics and Gynecology, 189, 494-500. http://dx.doi.org/10.1067/S0002-9378(03)00491-5 
[20] Gohil, J.T., Patel, P.K. and Gupta, P. (2011) Estimation of Lipid Profile in Subjects of Preeclampsia. The Journal of Obstetrics and Gynecology of India, 61, 399-403. http://dx.doi.org/10.1007/s13224-011-0057-0

[21] Clausen, T., Djurovic, S. and Henriksen, T. (2001) Dyslipidemia in Early Second Trimester Is Mainly a Feature of Women with Early Onset Pre-Eclampsia. British Journal of Obstetrics and Gynaecology, 108, 1081-1087. http://dx.doi.org/10.1016/s0306-5456(01)00247-9

[22] Cunningham, F.G., Leveno, K., Bloom, S., Hauth, J., Gillstrap III, L. and Wenstrom, K. (2005) Williams Obstetrics. McGraw-Hill, New York, 764-781.

[23] Palles, C., Johnson, N., Coupland, B., Taylor, C., Carvajal, J., Holly, J., et al. (2008) Identification of Genetic Variants That Influence Circulating IGF1 Levels: A Targeted Search Strategy. Human Molecular Genetics, 17, 1457-1464. http://dx.doi.org/10.1093/hmg/ddn034

[24] Mitsui, T., Masuyama, H., Eto, E., Nobumoto, E., Hayata, K., et al. (2015) Different Fetal and Neonatal Growth between Early- and Late-Onset Preeclampsia. Open Journal of Obstetrics and Gynecology, 5, 516-521. http://dx.doi.org/10.4236/ojog.2015.59074

[25] Barker, D.J.P. (2004) The Developmental Origins of Adult Disease. Journal of the American College of Nutrition, 23, 588S-595S. http://dx.doi.org/10.1080/07315724.2004.10719428

[26] Innes, K.E., Marshall, J.A., Byers, T.E. and Calonge, N. (1999) A Woman’s Own Birth Weight and Gestational Age Predict Her Later Risk of Developing Preeclampsia, a Precursor of Chronic Disease. Epidemiology, 10, 153-160. http://dx.doi.org/10.1097/00001648-199903000-00012

[27] Huxley, R.R., Shiell, A.W. and Law, C.M. (2000) The Role of Size at Birth and Postnatal Catch-Up Growth in Determining Systolic Blood Pressure: A Systematic Review of the Literature. Journal of Hypertension, 18, 815-831. http://dx.doi.org/10.1097/00004872-200018070-00002

[28] August, P., Baha, S., Charles, J. and Vanessa, A. (2011) Clinical Features, Diagnosis, and Long-Term Prognosis of Pre-Eclampsia. UpToDate Inc.

[29] Bravo, P.E., Morse, S., Borne, D.M., Aguilar, E.A. and Reisin, E. (2006) Leptin and Hypertension in Obesity. Vascular Health and Risk Management, 2, 163-169. http://dx.doi.org/10.2147/vhrm.2006.2.2.163

[30] Iftikhar, U., Khoja, A., Mehjabeen, I.A. and Karira, K.A. (2008) Evaluation of Serum Leptin Levels during Normal Pregnancy and in Pree-Clampsia. Journal of Ayub Medical College Abbottabad, 20, 137-140.

[31] Zhang, Y., Proenca, R., Maffei, M., Barone, M., Leopold, L. and Friedman, J.M. (1994) Positional Cloning of the Mouse Obese Gene and Its Human Homologue. Nature Clinical Practice Nephrology, 372, 425-432. http://dx.doi.org/10.1038/372425a0

[32] Friedman, J.M. and Halaas, J.L. (1998) Leptin and the Regulation of Body Weight in Mammals. Nature Clinical Practice Nephrology, 395, 763-770. 